\title{
Trabajo a domicilio en Chile: nuevas configuraciones de una antigua forma de trabajo de las mujeres
}

\author{
Claudia A. González \\ Universidad de Chile. Facultad de Ciencias Sociales \\ clau_andrea16@yahoo.com
}

Recepción: 02-01-2019

Aceptación: $18-03-2020$

Publicación: 22-05-2020

\section{Resumen}

Este artículo se centra principalmente en las formas de control y respuesta-resistencia de las trabajadoras a domicilio en el sector de la manufactura, específicamente en los sectores textil y de elaboración de sobres de papel en el Chile neoliberal. La metodología utilizada fue básicamente cualitativa, mediante la realización de entrevistas a distintos actores junto al procesamiento de encuestas a hogares. Respecto a los hallazgos, destacamos la funcionalidad del $\mathrm{TaD}$ en la preservación del desempeño de las labores de cuidado y tareas domésticas. En referencia a los registros de control, estos ocurren de manera articulada entre formas procedimentales y subjetivas. En un primer sentido, destacan el «trabajo a trato» y el "pago a destajo" como formas de control y sujeción al trabajo y, en una segunda dirección, los relatos de autonomía en la determinación de los tiempos de trabajo, que estimamos relativos y que en definitiva obscurecen la precariedad y la explotación que caracterizan estas formas de trabajo, persistentemente feminizadas.

Palabras clave: neoliberalismo; flexibilización del trabajo; trabajo a domicilio; control del trabajo; división sexual del trabajo; Chile 
Abstract. Home work in Chile: new configurations of an ancient form of women's work

This article centrally refers to the forms of control and response-resistance of homeworkers in the manufacturing sector, specifically in the textile and paper envelope manufacturing sectors in Chile. The methodological strategy wasqualitative, by conducting interviews with different actors. The process was accompanied by the analysis of household surveys. Regarding the results, we highlight the functionality of the $\mathrm{TaD}$ in preserving the performance of care work and domestic tasks; and as for the control registers, these occur in an articulated way between procedural and subjective forms; standing out in a first sense, the so-called "work to deal» and "piecework", as forms of control and subjection to work; and in a second, stories of autonomy in determining working times, which we consider relative; and that they ultimately obscure the precariousness and exploitation that characterize these persistently feminized forms of work.

Keywords: neoliberalism; work flexibility; work at home; work control; sexual division of labor; Chile

\section{Sumario}

1. Introducción

2. Enfoque e instrumental metodológico

3. Antecedentes del trabajo a domicilio en América Latina y en Chile

4. Configuraciones del control del trabajo de las trabajadoras domiciliares en contextos de flexibilidad productiva
5. Hallazgos: reconfiguraciones del trabajo domiciliar y formas de control y respuesta-resistencias en el $\mathrm{TaD}$ hoy en Chile

6. Conclusiones

Agradecimientos

Referencias bibliográficas

\section{Introducción}

Mediante este texto, esperamos acudir al lugar y al tiempo de una forma de trabajo precario de las mujeres que es muy antigua. Nos referimos al trabajo a domicilio (en adelante TaD). La Organización Internacional del Trabajo (OIT) define el trabajo a domicilio como la producción de bienes o prestación de servicios a un tercero. El trabajo se efectúa fuera de la empresa, generalmente en el hogar del propio trabajador/a, y no existe fiscalización directa por parte del empleador (Tomei, 1999). Si bien la OIT define el TaD en una condición de dependencia laboral, esta forma de trabajo también acontece en una modalidad de independencia, ya que en muchos casos las trabajadoras alternan o complementan ambas. Es un trabajo que se caracteriza por su composición preferentemente femenina y por ser altamente precario, a causa de su temporalidad, sus bajos e inestables salarios — que además son a destajo, es decir, se paga según lo producido- e insuficiente protección social y legal (Egan, 2019; Palacios et al., 2015; Brega et al., 2017; Leite et al., 2017). El $\mathrm{TaD}$ existe en el mundo desde los inicios del capitalismo y la industria (Tomei, 1999; Montero, 2000; Henríquez et al., 2005). En Chile, Elena Caffarena, ya en el año 1924, se preguntaba por las condiciones de vida y trabajo de las 
mujeres que desempeñaban estas labores (Caffarena, 1924). En la actualidad, el trabajo a domicilio persiste, pero en condiciones y modalidades distintas, ya no en contextos de industrialización, bajo modelos de producción centralizada de tipo taylorista-fordista, sino en contextos de flexibilidad, reorganización de la producción y el trabajo, y en una economía cada vez más globalizada (Henríquez et al., 1998; Leite et al., 2017). Este contexto de flexibilidad productiva, junto con la precarización del trabajo (a contrapelo del discurso de una supuesta modernización, mayor productividad y conveniencia para el trabajador), cuestiona las formas tradicionales de ejercicio del control del trabajo. El capital, en contextos de alta competitividad y globalización, demanda resituar y diversificar los registros y las formas de control. La pregunta base de la investigación que aquí exponemos es identificar e interpretar cuáles son y cómo operan las formas de control y resistencia de las trabajadoras a domicilio en el sector de la manufactura, específicamente en confección textil y de sobres de papel, vinculado a pequeñas, medianas y grandes empresas. Hipotetizamos que los mecanismos de control y respuesta-resistencia se configuran en un doble registro, procedimental y subjetivo, que opera de manera articulada, en tanto que el control procedimental requiere determinadas subjetividades, las que, a su vez, se sustentan en ciertas formas de organización de los procesos de trabajo.

Esta investigación se sitúa y se nutre de los estudios sociales del trabajo, con un enfoque de género e interseccional, y su aporte principal se refiere al análisis de un control articulado respecto al trabajo domiciliar. Además, esta modalidad de trabajo forma parte de encadenamientos de producción, en algunos casos globales, ${ }^{1}$ significativamente feminizados y con un entramado de relaciones de explotación y dominación que deviene, entre otros aspectos, en el doble desempeño, por parte de las trabajadoras, de las labores de producción y reproducción, las cuales acontecen en un mismo tiempo y espacio.

En el ámbito formal, el texto comprende, además de esta introducción, un segundo ítem donde se expone la metodología utilizada. En el tercero, se presentan antecedentes del TaD en América Latina y en Chile. En el cuarto, se propone un entramado conceptual que permite abordar los temas del control del $\mathrm{TaD}$ en los nuevos contextos. En el quinto, se presentan los principales hallazgos de investigación. Finalmente, en un espacio de conclusiones, se problematizan dichos hallazgos, atendiendo especialmente a la pregunta anteriormente planteada.

1. Cabe señalar, a propósito de los encadenamientos, en especial en confección textil, una nueva forma de producción, el llamado fast fashion, donde el $\mathrm{TaD}$ persiste, pero vinculado a cadenas globales de producción que polarizan el trabajo de diseño y producción, lo que redunda en la desresponsabilización de las condiciones de trabajo, especialmente de la producción, que ocurren en países de América Latina y el sur de Asia. Estas cadenas se caracterizan por una producción variada, de bajo coste, que cambia constantemente según las nuevas tendencias, y donde la producción a pequeña escala es del todo conveniente por los tiempos de producción y las fluctuaciones del mercado (Leite, et al., 2017). Sin embargo, de acuerdo con la CEPAL-OIT (2016), la participación de América Latina en encadenamientos globales de suministro es baja, en comparación con otros países del mundo, y se caracterizaría por una inserción con bajo valor agregado. 


\section{Enfoque e instrumental metodológico}

Abordar el análisis de la experiencia de trabajo de las mujeres y, más específicamente, la del control del trabajo, en sus registros procedimental y subjetivo, y desde su lugar de ocurrencia — que en nuestro caso coincide con el lugar donde acontecen otros ámbitos de la vida, como son las labores domésticas y de reproducción social—, nos conducía ineludiblemente a considerar una perspectiva de género. En ella, además, incluimos el concepto de interseccionalidad, dada la confluencia de más de un eje de desigualdad en las experiencias de vida y trabajo de las trabajadoras domiciliares, como son los ejes de clase, género, etarios o bien de origen. ${ }^{2}$ La definición de género propuesta por Domínguez et al. (2012) es sugerente, en el sentido de constituir una categoría de análisis no solo crítica y transformadora, sino también vinculada a debates actuales abocados a la comprensión de las diferencias y las desigualdades, situadas en un entramado social complejo (Zapata et al., 2013). Scott (2008) destaca que el género es un medio para investigar las formas específicas de la organización social de las diferencias sexuales. En esta investigación, mediante el uso de este enfoque, apostamos por la comprensión de la condición específica del trabajo de las mujeres que se desempeñan en el domicilio, de manera tercerizada, y donde confluyen la explotación del trabajo y la dominación de las relaciones de género. Según Roldán (1992), nada es impoluto al género: "Junto a cada división social y técnica del trabajo imbrica una división genérica del trabajo o segregación ocupacional por género» (p. 87). La noción de interseccionalidad nos permitirá analizar la combinación y la sinergia de distintos ejes de desigualdad y discriminación de manera integrada, pero además, como señala Caggiano (2014), en un contexto temporal y espacial concreto. La «intersección no es una sumatoria o convergencia simple de vectores, sino un proceso de configuración recíproca (Stolcke, 1992; Moore, 1993), vinculado a situaciones históricas concretas y contextos específicos (Yuval-Davis, 2011; Wade, 2008 y 2009)» (Caggiano, 2014: 154).

En lo que se refiere al instrumental utilizado, fue básicamente cualitativo. La razón de esta elección se sustenta en la posibilidad brindada por lo cualitativo de abordar la pregunta y el objeto definidos desde la perspectiva de las propias sujetas investigadas. En concreto, se realizaron 14 entrevistas semidirectivas $^{3}$ a trabajadoras de distintos sectores manufactureros, específicamente en la confección de vestuario y en la de sobres de papel, en este último caso para empresas mineras e imprentas. ${ }^{4}$ Destacamos la inclusión de ramos distintos al

2. El origen o lugar de procedencia es un aspecto presente en la literatura (Baylina, 1996; Egan, 2019; Kabat y Pascucci, 2010; Caggiano, 2014), y tambien referenciado en nuestras entrevistas, en el sentido de que existirían inmigrantes que trabajarían en casa. Sin embargo, en concreto, no se entrevistó a trabajadoras migrantes.

3. Donde se cuenta con una pauta de temas relevantes, más que con un guion de preguntas directrices (Valles, 1999).

4. De las 14 trabajadoras, dos eran dirigentes de organizaciones de trabajadoras a domicilio «de hecho", —dada la imposibilidad de constitución formal, por el desconocimiento legal de estas formas de trabajo en Chile- y otras tres trabajadoras externalizaban trabajo a vecinas. Se tuvo ocasión de entrevistar también a una subcontratista de sobres de papel. 
textil, dado que, al menos en Chile, se ha investigado preferentemente el trabajo domiciliar en dicho sector. ${ }^{5}$ Las trabajadoras entrevistadas fluctúan entre los 40 y los 60 años, viven en distintas comunas de la Región Metropolitana, ${ }^{6}$ cuentan con un nivel de educación secundaria, sus ingresos varían durante el año, pero no sobrepasan el ingreso mínimo de promedio, ${ }^{7}$ algunas se vinculan a encadenamientos que implican el eslabón de la gran empresa, pero la mayoría trabaja para pequeñas y medianas empresas. Algunas también se desempeñan simultáneamente por cuenta propia, en ventas esporádicas de prendas confeccionadas en ferias (mercados locales), como manera de complementar ingresos. En la mayoría de los casos, realizamos las entrevistas en los propios domicilios de las trabajadoras, para aproximarnos a las condiciones de trabajo y de vida de ellas y de sus familias; con la evidencia de la coconcurrencia de lo productivo y lo reproductivo en un mismo tiempo y espacio. Cabe señalar que además fueron entrevistados 14 informantes clave. El contenido de las entrevistas transcritas fue analizado mediante el programa Atlas ti y se definieron, previa e inductivamente, una serie de categorías constitutivas de nuestro objeto. ${ }^{8}$ Para la identificación de la muestra, se partió de algunos contactos disponibles, correspondientes al eslabón de trabajadoras, ${ }^{9}$ y a partir de los primeros contactos se utilizó la técnica llamada bola de nieve, que consiste en que una entrevistada deriva a otros. De manera complementaria, cabe agregar que se aplicaron técnicas cuantitativas, a través del procesamiento estadístico de las bases de datos de hogares de la Encuesta de Caracterización Socioeconómica (CASEN), de los años 90 y de 2013, con el objetivo de cuantificar el TaD durante un período determinado. Por último, destacamos la consulta y el análisis de una serie de fuentes secundarias de manera transversal a la investigación, con el fin de nutrir y problematizar teóricamente los hallazgos.

5. Entre las investigaciones desarrolladas en Chile, destacan las realizadas por la Dirección del Trabajo, del Ministerio del Trabajo, en los años 90', que se abocan principalmente a la caracterización del trabajo domiciliar. También cabe aludir a las investigaciones de Henríquez et al. (1998), Arteaga et al. (2003), Tomei (1999), Reinecke (1997) y Martini (2001), centradas en la confección de ropa, y Agacino et al. (1993), en el sector del calzado.

6. Las comunas equivalen a las unidades administrativas de menor tamaño, y la Región Metropolitana (R. M.) es donde se circunscribe Santiago, la capital del país. La opción de atender solo la R. M. se basa en la concentración del $\mathrm{TaD}$ en las zonas urbanas, entre las que destaca la Metropolitana, y las dificultades de cubrir otras regiones y comunas del país, en términos de financiamiento y tiempo.

7. El ingreso mínimo en Chile en la actualidad es de 301 mil pesos, que equivalen a 330 euros.

8. Como categorías, destacamos: Aspectos genéricos del TaD y de las trabajadoras (edad, trayectorias laborales, composición familiar, etcétera), Tiempos y espacios de trabajo, Mecanismos de control y Formas de resistencia.

9. Obtenida gracias al apoyo del Fondo Alquimia, la Confederación Contextil y la Oficina Municipal de Intermediación Laboral (OMIL), de la Municipalidad de Macul. El Fondo Alquimia es un fondo de mujeres que en el año 2014 coordinó el proyecto «Construyendo una agenda de los derechos laborales de las mujeres trabajadoras domésticas y de la costura en el Cono Sur», financiado por el Fondo Igualdad de ONU Mujeres y el Fondo Holandés FLOW. 


\section{Antecedentes del trabajo a domicilio en América Latina y en Chile}

$\mathrm{El} \mathrm{TaD}$ es una forma de trabajo de larga trayectoria que en la actualidad existe en condiciones y modalidades distintas, ya no en contextos de industrialización, bajo modelos de producción centralizada de tipo taylorista-fordista, sino que ocurre en situaciones de flexibilidad, reorganización de la producción y el trabajo y en el marco de una economía cada vez más globalizada. Forma parte de estrategias de fragmentación y desconcentración productiva que adoptan las empresas (Palacios et al., 2015) y que implican el traslado de los riesgos y costes de producción a los trabajadores (Egan, 2019; Kabat y Pascucci, 2010). El trabajo a domicilio se plantea como una condición de competitividad para las empresas (Henríquez et al. 1998). La producción externa reduciría costes internos e incrementaría la capacidad de respuestas ante los cambios en la demanda (OIT, 2001).

Un antecedente histórico del trabajo a domicilio fue el putting out system, un sistema de trabajo donde el dueño del capital aportaba las materias primas a trabajadores rurales que elaboraban en sus casas, de manera manual y discontinua, bienes con sus hiladoras y telares. Era un sistema doméstico útil al capital, ya que, al igual que hoy, otorgaba flexibilidad ante las fluctuaciones de la demanda (Martínez Galarraga, s/f). Con posterioridad a esa primera modalidad, el TaD acompañó los procesos de industrialización capitalista, donde la producción a gran escala concentró la fuerza de trabajo y los medios de producción en un mismo espacio, la fábrica, sin embargo, mantuvo las actividades manufactureras a pequeña escala realizadas fuera de la empresa, de manera que los talleres y el trabajo domiciliar constituyeron una extensión de las empresas.

En América Latina, el $\mathrm{TaD}$ como trabajo artesanal destinado a sectores modernos de la economía ha coexistido «con las formas más fordistas de organización de la producción» (Montero, 2000: 3). En contextos de flexibilización, el $\mathrm{TaD}$ se mantiene por su funcionalidad a las fluctuaciones del mercado y la generación de plusvalía, aunque con pesos específicos en las fuerzas laborales en cada uno de los países, medición relativa dada la dificultad y el subregistro en las encuestas nacionales (Tomei, 1999; Montero, 2000). En Brasil, el 3,9\% de la PEA para el año 1995 equivalía a trabajadores a domicilio; en Chile, al 1,5\% para el 1997, y en Perú, al $2 \%$ para el año 1993 (Tomei, 1999). ${ }^{10}$ En Chile, como señalamos, Elena Caffarena, ya en el año 1924, se preguntaba por las condiciones de vida y trabajo en particular de las mujeres que desempeñaban estas labores (Caffarena, 1924). En un estudio titulado «El trabajo a domicilio», publicado en el Boletín de la Oficina del Trabajo, del Ministerio de Industria y Obras Públicas de la época, la autora se refiere a estas formas de trabajo como «sweating system», analiza las condiciones de vida de las trabajadoras y sus familias y da cuenta de la profunda precariedad en la que trabajaban y vivían a propósito de los bajos salarios, las largas jornadas

10. Cabe señalar que no se dispone de datos más actualizados por la falta de mediciones específicas, lo que evidencia la invisibilidad económica y social de estas formas de trabajo, que se traduce, a su vez, en desprotección laboral y social de estas trabajadoras. 
de trabajo y las condiciones de hacinamiento e insalubridad de los hogares (Caffarena, 1924). En la década de los años 50, Walker Linares (1957) también refiere las condiciones de explotación asociadas a este trabajo y sostiene que estas formas de trabajar escapan a cualquier control y son una plaga del industrialismo moderno (citado por Henríquez et al., 2005). En los años 90, se realizaron una serie de estudios que cuantificaron y analizaron el trabajo a domicilio. Fueron análisis de casos de barrios o comunas, y en sectores específicos como la confección y el calzado (Henríquez et al., 1998). Un hallazgo común de estas investigaciones fue la precariedad de las condiciones laborales, que se expresaba en bajas remuneraciones y en ausencia de seguridad social. Igualmente, se constataría la preeminencia de las actividades de producción de bienes, largas jornadas de trabajo y altas exigencias de productividad y calidad, y el doble desempeño, por parte de las mujeres, tanto de las labores productivas como reproductivas (Henríquez y Riquelme, s/f). En el año 1997, para cuantificar el $\mathrm{TaD}$ en Chile, el Ministerio del Trabajo y el Instituto Nacional de Estadísticas (INE) agregaron a la Encuesta Nacional de Empleo (ENE) una encuesta suplementaria aplicada a una muestra de 34.000 viviendas a lo largo del país. Los resultados indicaron la existencia de 79.740 trabajadores a domicilio, un 1,5\% de los ocupados; el 82,3\% mujeres, equivalente al 3,9\% del empleo total femenino; un $96,4 \%$ de ellas localizadas en espacios urbanos, principalmente en las regiones Metropolitana, V y VIII. Respecto al tipo de trabajo que desempeñaban, destacaron la promoción y venta de servicios y la confección de prendas o partes de prendas, con más menos 35.000 y 15.000 personas respectivamente (Henríquez et al., 1998). Esta misma encuesta suplementaria se aplicó en el año 2000, pero esta vez solo en la Región Metropolitana. Los resultados mostraron un incremento del sector servicios y ventas, especialmente el porcentaje de trabajos profesionales como consultorías, asesorías, contabilidad, etcétera, que aumentó de un $10 \%$ en el año 1997 a un $25 \%$ en el año 2000; y disminuyó el porcentaje de mujeres, de un 80,2 \% a un 72,8 \% en los mismos años (Henríquez et al., 2005). Cabe señalar que se reconocen situaciones de subregistro, ya sea por posibles contextos de irregularidad del trabajo o de los trabajadores o bien porque en el momento de ser consultados no admiten su desempeño en el TaD por considerarse una ocupación secundaria, ante otras principales (Henríquez et al., 1998; Tomei, 1999). Después de esta fecha no se han realizado nuevas mediciones, sin embargo, en el marco de esta investigación, tras una actualización de estos datos, procesamos la base de datos de la CASEN ${ }^{11}$ del año 2013 y calculamos que 123.487 trabajadores

11. La encuesta CASEN ofrece información periódica de personas y hogares acerca de pobreza, datos demográficos, educación, salud, vivienda, trabajo e ingresos. En estas encuestas es posible identificar el lugar de trabajo, en nuestro caso, domicilios y talleres aledaños a él, según género. La CASEN del 1990 corresponde al primer año en que la encuesta fue aplicada, y la del 2013, al último año en el que se consultó por el lugar de trabajo, donde se consideran como categorías de respuesta "en el propio domicilio" y "en taller aledaño». La CASEN se realiza cada dos años, pero en las dos últimas, las de 2015 y 2017, se omitió la pregunta sobre el lugar de trabajo. 
se desempeñan en sus domicilios, de los cuales el 58,4 \% eran mujeres y el 41,67 \% hombres, un $44 \%$ de ellos en la Región Metropolitana. A su vez, para reconocer tendencias, acudimos a la CASEN del año 1990, que equivale al primer año de realización de esta encuesta. De acuerdo con los resultados para dicho año, 125.582 trabajadores se desempeñaban en sus domicilios, de los cuales un $52 \%$ eran mujeres y un $56 \%$ de la Región Metropolitana. Es decir, comparando ambos años, el total de trabajadores no varía significativamente, pero la composición según género varía en un $6 \%$ en favor de las mujeres, y los trabajadores ubicados en regiones distintas a la Región Metropolitana crecen en un $12 \%$. Cabe aclarar que no es posible comparar estos últimos datos con los de la encuesta de empleo suplementaria aplicada por la Dirección del Trabajo y el INE en la década de los años 90 y luego en el 2000 (Henríquez et al., 1998; Henríquez et al., 2005), ya que aquellos resultados se refieren a otras bases, específicamente las de empleo. A diferencia de la CASEN, que es una encuesta de caracterización socioeconómica de hogares; en definitiva, son muestras e instrumentos de medición distintos.

Por último, nos referimos a un aspecto de significación en la caracterización del $\mathrm{TaD}$, equivalente a la legislación que lo reconoce y protege o, en su defecto, lo obvia y precariza. En América Latina existen diferencias entre los países, ya que algunos cuentan con leyes especiales que lo reglamentan, como es el caso de Argentina; o bien con un código laboral con orientaciones específicas para este tipo de trabajo, como en Paraguay y Perú, y en otros, como Brasil, los derechos de las trabajadoras domiciliares son comunes al resto de fuerza laboral (Tomei, 1999). En Chile, el TaD se reguló por primera vez en el año 1931, como parte del primer código del trabajo, que admitía estas prestaciones como «una especie de contrato de trabajo» (Olate, 1995, citado por Henríquez et al., 1998). Después, en el marco del Plan Laboral aprobado durante la dictadura cívico-militar encabezada por Pinochet, se derogó el párrafo que regulaba y reconocía el contrato de trabajo a domicilio. Durante el primer gobierno de transición democrática, en la década de los años 90, se contempló la reposición de la calificación de «contrato de trabajo», iniciativa que no prosperó. En Chile, el contrato que rige la relación es de una prestación de servicios regida bajo la ley civil (Henríquez et al., 1998). Esta negación del vínculo laboral y su reemplazo por un vínculo de servicios constituyen un aspecto de total precarización, característico del trabajo a domicilio y de otras numerosas formas de trabajo en el Chile actual, por ejemplo, todas aquellas vinculadas a aplicaciones de internet, cuyo incremento resulta evidente en la cotidianidad del país.

\section{Configuraciones del control del trabajo de las trabajadoras domiciliares en contextos de flexibilidad productiva}

Los procesos de reconfiguración y tercerización productiva han significado la ampliación o la difuminación de las fronteras de la empresa, además de la alteración de los tiempos y lugares de trabajo y la emergencia de nuevas formas de control (Maza, 2004; Alonso y Fernández Rodríguez, 2009). Según 
Todaro y Yáñez (2004), en contextos de una nueva organización productiva, el control directo o externo del trabajo es reemplazado por uno indirecto o interno «que define solo los objetivos, metas y recursos disponibles, dejando a la discrecionalidad del trabajador la manera de organizar su trabajo (...)» (Todaro y Yáñez, 2004: 70).

En términos genéricos, es posible reconstruir una trayectoria o identificar distintas formas que el control ha adoptado a lo largo de la historia del trabajo. En la época preindustrial, este control era responsabilidad o atributo del artesano u obrero de oficio. Posteriormente, las demandas del capital y su acumulación exigieron el traspaso del ejercicio del control del trabajador al capitalista; para garantizar el uso pleno de la capacidad de la fuerza de trabajo adquirida (Garza, 2011). Ese habría sido precisamente uno de los objetivos del taylorismo, la enajenación del saber propio del oficio, la descalificación y apropiación del control de los tiempos de producción. Según Coriat (2011), una de las principales innovaciones de la organización científica del trabajo - promovida por Taylor - era precisamente reemplazar el control obrero del proceso de trabajo mediante la imposición de un "conjunto de gestos» de producción concebidos y preparados por la dirección de la empresa y cuyo respeto es vigilado por ella (Coriat, 2011: 36). Es lo que se nombró one best way, que tenía como objetivo acabar con el oficio y el control obrero respecto a los tiempos de producción (Coriat, 2011). La generalización del taylorismo en las primeras décadas del siglo xx y la implementación de la «organización científica del trabajo» constituyeron hitos centrales en la historia del dominio patronal y la acumulación del capital (Katz, 2000). Así se conformó la producción taylorista en el paradigma del control del trabajo. El taylorismo predominó durante toda una etapa de capitalismo de acumulación extensiva y alcanzó niveles importantes de productividad, pero finalmente este modelo se agotó.

Hoy, en contextos de flexibilidad productiva, el control directo y externo adopta formas indirectas e internas, donde el trabajador (supuestamente) dispondría de cierta autonomía en la organización del trabajo y donde se promueven «formas internalizadas de autocontrol, autoorganización y autorracionalización por parte de los mismos trabajadores» (Todaro y Yáñez, 2004: 70). Como señalan Alonso y Fernández Rodríguez (2009), en las empresas se instala la consigna del compromiso y la participación del trabajador, «para ello es imprescindible que esté socializado en un sistema de valores que le hagan trabajar al máximo, aunque no se le vigile» (Alonso y Fernández Rodríguez, 2009: 5). De acuerdo con Narbona, «se exige al trabajador una compenetración emocional y moral con el trabajo, más allá del cumplimiento del contrato. Un compromiso arraigado en orientaciones personales e íntimas, que lo hagan más proclive a la adaptación permanente, a la mejora continua y al esfuerzo adicional» (Narbona, 2012: 5).

En el caso del trabajo domiciliar, las formas de control históricamente han diferido de las formas propias de la industria, en las cuales el empleador supervisaba directamente la cantidad, la calidad, la maquinaria, los métodos de trabajo, las calificaciones, etcétera (Garza, 2010). En el TaD, en cambio, el 
control es generalmente intermediado por el subcontratista, quien se encarga de distribuir, recibir y supervisar la calidad y los tiempos del trabajo, y de descontar salario por atraso y piezas defectuosas (Abramo et al., 2004). El control se efectúa ex-ante a la realización del trabajo, cuando el contratista especifica las características del bien a producir, y después de su ejecución, al verificar el cumplimiento de lo exigido (Tomei, 1999). También es posible que el empleador determine los tiempos, la calidad y los valores de lo producido, o bien el contratista fija el precio a pagar a las trabajadoras y negocia con la empresa el precio de la prenda (Arteaga et al., 2003). En definitiva, el trabajo a domicilio, a pesar de su condición de externalidad respecto a la empresa, sigue bajo su lupa, ya que esta mantiene el control sobre la producción, las tarifas y los plazos de entrega (OIT, 2001), solo que de manera indirecta, mediante los contratistas, y no en el espacio y los tiempos de la fábrica, sino que en los domicilios y en los tiempos de vida de las trabajadoras y sus familias.

Como mecanismo clave de control y sujeción al trabajo en el TaD, también cabe destacar la determinación de la tarifa y las formas de salario, que son a destajo, es decir, se paga según lo que se produce. Para Egan (2019), «el pago a destajo ata el salario del obrero a la cantidad de prendas producidas, por lo que lo obliga a autoexigirse para aumentar los ritmos y, por ende, la cantidad de producción» (p. 5). Esta forma de salario, al igual que el TaD, existe desde hace mucho tiempo, pero persiste en procesos flexibles de producción y configura formas de control que reemplazan el control directo del empleador (Sevilhano Martínez, 2008).

$\mathrm{El} \mathrm{TaD}$, por su deslocalización respecto a la empresa, no solo requiere, sino que además constituye un terreno fértil para la instalación de dispositivos de control que aluden al autocontrol y la autoorganización del trabajo. Estos últimos aspectos son característicos de formas de producción flexibles (Todaro y Yánez, 2004). Las trabajadoras domiciliares, en tanto trabajo vivo, siempre contaran con la discrecionalidad de consentir o resistir el trabajo. Es por ello que el capital requiere acoplar a las formas de control operacionales formas subjetivas que garanticen la ejecución del trabajo en tiempo y forma, y que estimamos, se traducen en discursos favorables a su desempeño por parte de las mujeres, como la mayor experticia de estas para realizarlo, su conveniencia porque les permite mantener las responsabilidades de cuidado y reproducción, la autonomía para determinar los tiempos de trabajo, etcétera, inclusive aquellos discursos del emprendimiento y el esfuerzo como condiciones sine qua non para el éxito de cualquier iniciativa que se adopte. Sin embargo, lo que acontece es que estas nociones de autonomía y emprendimiento se instalan como discursos hegemónicos y formas de control subjetivas que más bien y principalmente solapan condiciones de explotación y dominación.

El control del $\mathrm{TaD}$ se configura en esta dualidad —operacional y subjetiva- y mediante relaciones sociales situadas ya no en la industria, sino en una empresa ampliada y flexible. Son relaciones de control y poder que muchas veces se dan en el marco de vínculos familiares o de vecindad, entre trabajadoras y contratistas, lo que hace más complejas las relaciones de trabajo. Sugerente 
es el aporte de Narotzky (1997), quien, en un estudio comparativo entre dos regiones de España e Italia de economía flexible, analiza la experiencia del trabajo y la construcción de identidades sociales. La autora reconoce que las relaciones de familiaridad o vecindad son más bien una vía de realización de la explotación, parecen una cosa pero son otra. Además, detrás del trabajo a destajo y el trabajo familiar, hay autoexplotación y explotación, extracción de plusvalía de la mano de obra familiar. Igualmente interesante es lo señalado por Borgeaud-Garciandía y Lautier (2014) en un estudio realizado en torno a las situaciones laborales de trabajadoras de la maquila y domésticas en América Latina. En él, se interroga acerca de la personalización de las relaciones de dominación presentes en este tipo de trabajos y se reconoce que las formas de dominación personalizada se sustentan en una doble ficción: una «ficción de generosidad» del que da empleo versus una «ficción de deuda» de quien lo asume. Además, esto se daría en un contexto de precariedad de opciones, «donde la dominación está fuertemente ligada a la idea de que las posibilidades de trabajar en otro lugar son extremadamente restringidas, incluso inexistentes...» (Borgeaud-Garciandía y Lautier, 2014: 108).

\section{Hallazgos: reconfiguraciones del trabajo domiciliar y formas de control y respuesta-resistencias en el $\mathrm{TaD}$ hoy en Chile}

A continuación, daremos cuenta de nuestros hallazgos de investigación en lo que se refiere a las configuraciones del $\mathrm{TaD}$, y en especial en torno a las formas de control y resistencias de las trabajadoras domiciliares. Cabe precisar que la investigación permitió identificar una serie de aristas interesantes referidas a las condiciones de vida y trabajo de las trabajadoras domiciliares, y también a las determinantes de género en las configuraciones no solo del oficio en el domicilio, sino también de sus trayectorias laborales. Sin embargo, para efectos de lo que aquí se expone, centraremos nuestra exposición en los aspectos del control, en sus diversos registros y también en sus resistencias.

\subsection{Cómo se ejerce y configura el control del trabajo en el TaD}

Uno de los mecanismos de control hallados y anticipados por la literatura corresponde al llamado pago a destajo. Es una forma de control indirecto que opera básicamente respecto a la cantidad de trabajo y tangencialmente respecto a la calidad. Sujeta a los individuos al trabajo y los implica intensa y extensivamente en términos temporales, con una remuneración por debajo del valor del trabajo, lo que deviene frecuentemente en dinámicas de autoexplotación y de superexplotación. Para Sotelo Valencia (2003), son tres los mecanismos fundamentales por los que se hace efectiva la superexplotación: la prolongación de la jornada reglamentaria, el aumento de la intensidad del trabajo y una remuneración de la fuerza de trabajo inferior a su valor. En definitiva, se trata de una apropiación del tiempo de trabajo necesario para su reconversión en fondo de acumulación (Salgado y Carpio, 2017: 66). En nuestro caso, concurren estas tres 
formas. El pago a destajo demanda el cumplimiento de determinados tiempos y cuotas de producción, formas que reemplazan el control directo o se acoplan a él. En este mismo sentido, Prates (1984) advierte que el pago por pieza es una manera de control no solo de la cantidad, sino también de la calidad. La autora hace referencia a Marx y Engels, quienes sostendrían que el pago a destajo, sostén del trabajo a domicilio, haría innecesario el ejercicio del control: "Como la calidad e intensidad del trabajo están controladas aquí por la forma misma del salario, esta vuelve superflua gran parte de la vigilancia del trabajo» (Marx y Engels, 2002: 2). Prates agrega que en el destajo el trabajo que se remunera es el que alcanza una cierta calidad. En caso de fallas en su confección, la pieza defectuosa y el tiempo invertido en su reparación no son remunerados, lo que coincide con los relatos de las trabajadoras entrevistadas. Ahí se señala que, al errar en la confección de la prenda, ellas asumen los costes de producción y el tiempo reinvertido no es remunerado: "Cuando hay falla en el trabajo, el subcontratista lo que hace es que (el trabajador) tiene que desandar, y no les vuelve a pagar el salario de la equivocación. Eso ya está pagado» (trabajadora de confección de ropa y dirigente). Las fallas cuestionarían además la sostenibilidad de la relación laboral, pero no se identificaron situaciones del cobro de multas por error, cuestión presente en la literatura (Leite, 2004). Otro aspecto a destacar es el efecto del pago a destajo sobre la relación empleador-trabajador. El destajo traslada la determinación del salario desde la relación trabajador-empleador y lo resitúa en el propio esfuerzo del trabajador. Según Menéndez (2015), ello no implicaría que los trabajadores no puedan negociar su salario - lo que en nuestro caso de todas maneras no ocurre-, sino que lo que acontece es más bien que «la variable de ajuste a la que cotidianamente recurren los trabajadores es la medida de su esfuerzo, que será puesta en cuestión solamente en aquellos casos en los que llega a percibirse como injusta o inaceptable la remuneración así obtenida" (Menéndez, 2015: 93). En el caso de algunas de las trabajadoras entrevistadas, estas renunciaban a acuerdos o tratos que ellas estimaban en extremo mal pagados, lo que identificamos como una ocasión de agencia y resistencia por parte de las trabajadoras.

Vinculado a lo anterior, en el sentido de la sujeción al trabajo y margen de negociación de las trabajadoras o, como plantean Edwards y Scullion (1987), la negociación del esfuerzo por parte de los trabajadores, cabe añadir un aspecto clave: la tarifa, que corresponde al pago por tipo de labor o prenda, cuestión ya presente en los tiempos de Caffarena y vigente hasta el día de hoy. Según registramos en las entrevistas realizadas, la "tarifa» no es un tema menor, ya que en la definición de las tarifas se determinan implícitamente también aspectos referidos a los tiempos y la calidad del trabajo (Coriat, 2011). En lo hallado, la tarifa generalmente se establece unilateralmente por el empleador o subcontratista: «Ni siquiera uno tiene la opción de decir no, yo quiero que me pague esto. No. Este es el precio que hay, y es lo que hay» (trabajadora de confección de ropa).

Respecto al control del proceso de trabajo, en el caso del $\mathrm{TaD}$, en un registro operacional o, en términos de P. Edwards (1990), detallado, cuenta 
con menor densidad en comparación con el ejercido en el interior de la fábrica fordista, donde existía una cadena de producción que parcelaba e intensificaba el trabajo y donde el empleador ejercía un control directo en cada una de las etapas del proceso de trabajo. ${ }^{12}$ En el $\mathrm{TaD}$, el control consiste en la entrega de orientaciones previas y la revisión del trabajo una vez finalizado, formas anticipadas por Tomei (1999) y corroboradas en esta investigación. El control es ejercido sobre la ejecución del trabajo — se prescinde de las etapas del diseño, dado que, en el caso de la confección de ropa en particular, se replica la práctica ya reportada en la literatura referida a la fragmentación de la producción-, ya que las trabajadoras reciben solo las piezas a manufacturar. El control se ejerce en la ejecución, pues las etapas de diseño y organización son responsabilidad del empleador o subcontratista. De esta manera, en el $\mathrm{TaD}$ se replican aspectos típicamente fordistas, en el sentido de la división entre concepción y ejecución del trabajo, escisión en la que se sustenta precisamente el control del trabajo (Braverman, 1983). También cabe precisar que la frecuencia de dicho control depende del tipo de confección. Según una de las trabajadoras entrevistadas vinculada a una gran empresa que comercializa ropa de niños, el subcontratista supervisaba el trabajo durante la confección por las condiciones particulares de la tela que se utilizaba.

Además, hay que destacar que el control es mediado por el subcontratista - no existe una supervisión directa del empleador-, quien, al comienzo, al final y, solo ocasionalmente, durante el proceso de trabajo, asiste al taller para controlar la calidad de la prenda. La presencia del contratista, y no la del empleador, se entiende en contextos de subcontrato y como parte de estrategias o dispositivos de control de las empresas, que, como señala Coriat (2011), utilizan a su favor la cercanía que existe entre el subcontratista y las trabajadoras y el mayor conocimiento de este en el hacer y en las condiciones de trabajo. En nuestras entrevistas, inferimos que en la mayoría de los casos el subcontratista descansa en el saber hacer de las trabajadoras, adquirido a lo largo de sus trayectorias laborales, lo que garantizaría el logro del producto y la calidad requerida. Es decir, son las trabajadoras las que, parafraseando el mandato taylorista, deciden "la mejor manera» de hacer el trabajo. Además, por el pago a destajo, la mejor forma será aquella que les permita cumplir la cuota e incrementar sus ingresos como consecuencia de una mayor producción. Reconociendo esta autonomía — relativa - por parte de las trabajadoras, en la medida de determinar el cómo, tiene sentido la distinción de Friedman (1977) en torno al control directo y la «autonomía responsable». «En el primer caso, los patrones ejercen un dominio autoritario e incuestionable; en el segundo, ellos delegan alguna posibilidad de decisión a los operarios y escuchan sus sugerencias, sin, con todo, poner en cuestión la estructura de comando y el sentido

12. P. Edwards distingue entre control detallado y control general. El primero contempla el control respecto a cada aspecto del proceso de trabajo, y el control general implica lograr «el compromiso de los trabajadores con los objetivos de la empresa» (Edwards P., 1990: 70). 
de la producción» (en Mello, 2014: 164). ${ }^{13}$ En las trabajadoras entrevistadas, estas definen autónomamente la mejor manera de hacer en la ejecución de tareas parciales. Sin embargo, no inciden en aspectos sustantivos, como es la definición de tarifas, tiempos y cuotas de trabajo.

En lo que se refiere a la dimensión subjetiva del control del trabajo, el capital históricamente ha acudido, o más bien ha promovido, a una serie de constructos culturales útiles en la sujeción al trabajo, los cuales constituyen un sentido común que configura un dispositivo disciplinador (Alonso y Fernández, 2009) que norma las configuraciones y los tiempos de trabajo. Estos constructos existen desde hace mucho tiempo, pues se hicieron ya presentes en los albores de la revolución industrial con la llamada ética del trabajo (Bauman, 1999), y se han reconfigurando a lo largo de la historia social y del trabajo de acuerdo con las necesidades del capital y las capacidades de respuestas de los distintos actores. En la actualidad, asumen configuraciones particulares a propósito del llamado modelo toyotista, con figuras como «calidad total» $\mathrm{y}$ «justo a tiempo», entre otras, donde se promueven trabajadores autónomos y comprometidos con los objetivos de las empresas. Como señala Álvarez (2012), a diferencia del taylorismo, donde se planteaba una mejor manera de hacer, hoy «el saber ser se impone al saber hacer» (p. 56). Es decir, se reconocen «dispositivos de control más sutiles que apuntan a formar a los trabajadores en una dialéctica de saber ser - saber hacer, que supone una misma lógica de formar para involucrarse en los objetivos de trabajo y formar para aumentar la productividad» (Álvarez, 2012: 49). Como decíamos anteriormente, se exige a los trabajadores un compromiso personal e íntimo que los lleve al ejercicio de un esfuerzo y una mejora continuos (Narbona, 2012). En las entrevistas, encontramos relatos que comprenden el compromiso tanto con el trabajo como entre trabajadoras y subcontratistas. Respecto del primero se dirá: «Porque es mi pega, mi trabajo, y yo siempre he dicho que si yo me comprometo a algo, es porque lo puedo hacer y lo voy a hacer bien, porque, si no, no lo tomo no más» (trabajadora de confección de sobres de papel). Del trabajador respecto del contratista: «Yo le voy a terminar sus sobres. Porque no se los voy a dar así, porque sería una falta de respeto" (trabajadora de confección de sobres de papel). La demanda de compromiso del subcontratista respecto de las trabajadoras puede adoptar expresiones profundamente personalizadas, como se evidencia en el siguiente relato:

Yo les agradezco eso (a las trabajadoras) porque han sido personas superresponsables, han valorado harto el trabajo, e igual eso me emociona harto, me emociona, encuentro superbonito que alguien, no sé, valore el trabajo, tenga esa afinidad, ese compromiso porque se comprometen conmigo (Subcontratista de confección de papel).

También ocurre que los subcontratistas manifiestan compromiso, en términos de la provisión del trabajo: «Siempre pienso que, si yo lo dejo, voy a dejar a la gente sin trabajo, y eso es lo que a mí me (...), porque hay gente que

13. El texto original estaba en portugués, la traducción es propia. 
depende de esto, por eso, que me da cosa que cuando no llega pega (trabajo), uno o dos días (...)» (subcontratista de confección de papel).

Estimamos que el discurso del compromiso es un mecanismo de sujeción al trabajo, un compromiso con un otro y también vinculado a un mandato social y ética del trabajo, a costa de dinámicas de autoexplotación. La distinción propuesta por Espinoza y Soto (2008), desde distintos enfoques de la psicología y la sociología de las organizaciones para comprender la implicación de las personas con su trabajo, es sugerente. Los autores distinguen entre la implicación afectiva, la de continuación y la normativa: la afectiva deriva de experiencias positivas que alientan y sostienen el vínculo con la empresa; la de continuación respondería a una decisión calculada de coste-beneficio, de continuar o mantenerse, y la normativa se sustenta en "procesos de socialización del individuo y en las creencias interiorizadas acerca de la lealtad que la persona deba demostrar ante una entidad» (Espinoza y Soto, 2008: 282). En nuestro caso, hallamos preferentemente dos de esas formas. La afectiva se da principalmente cuando las relaciones existen desde hace mucho tiempo o bien hay vínculos familiares. La normativa está vinculada a lo ya mencionado de la presencia de una cierta ética del trabajo, de responsabilidad y compromiso. Respecto a la de continuidad, esa se ve alterada cuando las trabajadoras estiman que la tarifa impuesta es muy baja. Godoy y Stecher (2008), por su parte, a propósito de un estudio de trabajadoras de servicios y comercio, destacan una cierta internalización del control, que se manifiesta en «lógicas de automonitoreo y autorregulación» (Godoy y Stecher, 2008: 201). En el caso de las trabajadoras entrevistadas, ellas conciben esta autorregulación como un requisito para el cumplimiento del trabajo, en el sentido de que de otra manera no sería posible.

Junto con las nociones de compromiso y autodisciplina, hallamos una serie de relatos favorables al $\mathrm{TaD}$. Es recurrente, por ejemplo, la afirmación acerca de la autonomía en la definición de los tiempos de trabajo, en el sentido de poder reorganizar de manera flexible sus jornadas diarias de trabajo en función de las exigencias tanto reproductivas como productivas. A diferencia de las rigideces horarias de la fábrica o del "trabajo típico» o formal extradoméstico, donde habría que cumplir jornadas fijas de trabajo, en el domicilio ellas reorganizarían sus jornadas de acuerdo con las rutinas y las eventualidades del cuidado y domésticas, es decir, determinan el qué y el cuándo de lo productivo y reproductivo según sus «necesidades»: «Puedes distribuir tu tiempo, tu carga de trabajo, en distintas horas» (trabajadora de confección de ropa).

Sin embargo, en el siguiente relato se evidencia la tensión que existe entre la autonomía en la determinación de los tiempos y la presión del comprador respecto a la entrega del trabajo:

Aquí yo tengo mi tiempo, soy yo la que controlo mi tiempo; yo puedo trabajar día y noche, día y noche, pero después me tomo un día completo, de repente tengo que trabajar aquí día y noche, porque, como trabajo sola, o 
de repente viene la señora Emilia y me ayuda, no me alcanza, entonces la gente empieza a apurarte, a apurarte, y te exige (Trabajadora de confección de ropa).

Al tensionar la realidad y la consistencia de este relato autonómico, evidenciamos su relatividad en varios sentidos. Por un lado, si bien las trabajadoras señalan definir autónomamente los tiempos de trabajo y disponer de flexibilidad para reorganizar las dobles jornadas, deben realizar la tarea en el marco de los plazos de entrega, determinados por los subcontratistas y en los cuales ellas no tienen ninguna incidencia. En caso de ser tiempos breves, tienen que comprometer jornadas intensas y extensas de trabajo e incluso incorporar a la familia para cumplir con la cuota y los tiempos establecidos. Por otro lado, como señalamos, las trabajadoras carecen de poder de decisión respecto a otros aspectos sustantivos en el proceso de trabajo, como son las cuotas de trabajo, las tarifas y, por ende, los salarios. Sin embargo, junto con cuestionar o colocar en entredicho el relato de autonomía en el manejo de los tiempos, en el sentido de lo aparente, o bien lo limitado, cabe reconocer que, tras la noción y (relativa) opción de manejar los tiempos cotidianos, se sucede cierta «humanización» del trabajo, en contraste con la condición de trabajo de la fábrica, donde, como nos señala una de las entrevistadas, «uno se vuelve como parte de la máquina». En la siguiente cita, esta trabajadora nos relata su experiencia en la fábrica y la contrasta con la realidad del domicilio:

Cuando entré a trabajar a Arrow, yo quedé impresionada. Te lo juro, porque no eran personas, eran máquinas, y yo después me puse igual que ellas (...), usted no podía pararse, porque era a trato, si quería sacar un buen sueldo tenía que matarse trabajando ahí (...), es increíble pero uno se vuelve como parte de la máquina en una fábrica. Porque aquí (en casa), si yo quiero, me paro; si quiero, veo televisión; si quiero, voy y me sirvo una taza de té y sigo trabajando, pero en la fábrica no (...), no volvería a trabajar en fábrica. Como le digo, uno se vuelve como un robot (Trabajadora de confección de ropa).

\subsection{Configuraciones (o formas) de resistencia}

La pregunta por la resistencia de las trabajadoras se sustenta en el reconocimiento de la capacidad de las personas de agencia y respuesta frente a situaciones, en este caso, de sometimiento a condiciones de trabajo precarias. Garza (2001) plantea que una acción no es siempre una acción heroica, ya que puede ser un acto de negociación e incluso de subordinación. En este caso, concebimos un registro de respuesta que puede transitar entre el consentimiento del trabajo, que se expresa a su vez en la reproducción de un relato social en sintonía con el compromiso y la responsabilidad frente al trabajo, a una expresión sindical o la constitución de organizaciones reivindicativas.

En las entrevistas realizadas, al tratar de dilucidar las acciones desplegadas por las trabajadoras frente al control en un registro de resistencia, encontramos que las trabajadoras renuncian a ciertos vínculos que estiman altamente 
precarizados, con bajo retorno económico, al considerar una alta inversión de trabajo, en búsqueda de otros que consideran más rentables. Otra forma de resistencia viene dada por el intento de organizarse como trabajadoras a domicilio y constituir organizaciones de hecho, sin contracara o respaldo legal, en la perspectiva de evidenciar la precariedad de sus trabajos y apostar por su reconocimiento como trabajadoras. Valoramos estas iniciativas, que se sustentan en una autorrepresentación como trabajadoras, con una agenda de reivindicaciones particular donde se destaca también el liderazgo de algunas de ellas, que han tenido previamente experiencias de trabajo y sindicales en la industria de la confección, lo que les permiten ejercer su liderazgo de manera ejemplar. Sin embargo, no ha sido un proceso de organización fácil de sostener. La dispersión física no les favorece, ni tampoco las dobles jornadas de trabajo, que hacen difícil disponer de tiempos extras. En la constitución de organizaciones de hecho, cabe destacar algunos aspectos importantes. Desde las organizaciones de trabajadoras a domicilio de hecho o de facto, se ha planteado la posibilidad de ser incorporadas en las negociaciones colectivas de los trabajadores de fábricas, lo que permitiría no solo visibilizar a las domiciliares, sino también resguardar las condiciones de trabajo de los internos mediante la homogenización de las condiciones de trabajo — entre internos y externalizados. Así, se obstaculizaría la práctica empresaria de externalizar la producción en contextos de conflictividad interna o inconveniencias en las negociaciones colectivas. Sin embargo, ello no ha sido posible por el temor de los trabajadores internos de debilitar sus demandas por la informalidad de las domiciliares y ha primado, en definitiva, el conflicto entre pares, en desmedro de conflictos con el empleador.

Como aspectos que dificultan la generación de respuestas en un registro de resistencia, también cabe señalar el desdibujamiento de las relaciones de trabajo por la informalidad y, vinculado a ello, la performatividad de una relación de trabajo que figura como de servicio. En otro sentido, un aspecto que también obstaculizaría la respuesta-resistencia de las trabajadoras es la cercanía entre trabajadores y subcontratistas, lo que hace compleja e impide la opción de conflicto.

Por último, un abordaje posible de las resistencias, en su variante organizacional y colectiva, se vincula a la construcción de identidades en el trabajo, que en el caso del $\mathrm{TaD}$ estarían doblemente tensionadas por el lugar y por la labor: un lugar asociado a lo doméstico y lo privado; y una labor, en particular en las trabajadoras de confección de ropa, asociada y naturalizada como típicamente femenina. En definitiva, la asignación cultural de roles y lugares terminaría cuestionando y dificultando la organización y las resistencias de las trabajadoras.

\section{Conclusiones}

La importancia de esta investigación se refiere a la posibilidad de problematizar respecto a la condición de trabajo y explotación de un sector de trabajadoras que se encuentran invisibilizadas social, económica y legalmente. La opción 
de las mujeres por el trabajo a domicilio se comprende en la intersección de una condición de clase que supedita las opciones de inserción en el trabajo y de generación de ingreso, junto con las condiciones y los determinantes de género que las sujeta a los espacios domésticos y al desempeño de las labores de cuidado. En el trabajo a domicilio concurren la explotación por el trabajo y la sujeción a lo doméstico y al cuidado; es decir, coinciden la explotación en el lugar recóndito de la producción y la dominación por la reproducción.

Mediante esta investigación se retomó una pregunta tradicional de los estudios del trabajo referida al control y aplicada a una forma de trabajo que existe desde hace mucho tiempo: el trabajo a domicilio. Se reinstaló en nuevos contextos de ocurrencia, ya no en los de la industria fordista, escenario original en el que surge este debate acerca del control del proceso de trabajo, sino en contextos de reestructuración y flexibilización del trabajo y la producción. El sentido era dilucidar las actuales formas de un trabajo muy antiguo, que en la actualidad acontece en los pliegues de una modernización global y excluyente, especialmente las configuraciones del control que allí se daban. Corroboramos la hipótesis planteaba en el sentido de que el control del $\mathrm{TaD}$ se configura en la articulación de un control que acontece en un doble registro, operacional y subjetivo. Que además ocurre en relaciones sociales situadas ya no en la industria sino en una empresa ampliada y flexible. Son relaciones de control y poder que muchas veces también se dan en el marco de vínculos familiares o de vecindad, entre trabajadoras y contratistas, lo que hace complejas las contradicciones clásicas de capital-trabajo, así como visiones esencialistas e idealizadas de las relaciones entre pares. El análisis de la experiencia articulada del control entre lo procedimental y lo subjetivo descansa en la premisa de que estas experiencias pueden ser comprendidas precisamente en esta interfaz, donde los procedimientos concretos de control tienen su expresión subjetiva. A su vez, el control subjetivo se encarna materialmente en procesos de trabajo y formas de control directo. Como señala Narotzky, es necesario atender «el campo ideológico en que toman cuerpo las relaciones de producción» (Narotzky, 1988), o bien «la hegemonía ideológica puede mantenerse solo si descansa sobre bases materiales» (Przeworski, 1988: 15). Controles directos e indirectos, procedimentales y subjetivos, que favorecen la acumulación del capital y garantizan la conversión del potencial de trabajo en trabajo real.

Destacamos, además, el uso de una perspectiva de género e interseccional, dada la presencia de una serie de representaciones y autorrepresentaciones que creemos que coadyuva a configurar las formas que adquiere el control en las trabajadoras a domicilio. Estimamos que las trabajadoras determinan autónomamente el cómo del trabajo, con base en la experiencia y saberes del trabajo, tras una mayor productividad y salario; así como también reorganizan la doble jornada, productiva y reproductiva, en el sentido de definir el qué y el cuándo realizan una u otra labor, pero sin desentenderse de ninguna.

En el ámbito productivo, definen el cómo, pero careciendo de decisión respecto al resto de aspectos sustantivos del proceso y las condiciones de trabajo, como los tiempos, las cuotas, la tarifa y, por ende, los salarios. Más bien depen- 
den de las exigencias y los criterios de los contratistas, ya que las trabajadoras, a condición de cumplir con la cuota y preservar el vínculo laboral, cumplen jornadas frecuentemente intensas y extensas de trabajo que implican, si es necesario, trabajo nocturno o de fin de semana, y con la colaboración de la familia. Es decir, la autonomía que señalan es parcial, relativa; además, se produce en el marco de una relación asimétrica y de poder que ha sido invisibilizada por la particularidad del nexo laboral — que figura como de servicios y no laboral—, y también por su irregularidad y por el carácter intermediado, que en la mayoría de los casos comprende relaciones de cercanía (vecindad o familiaridad) entre trabajadores y subcontratistas. En los ámbitos reproductivos, si bien atestiguamos nuevos acuerdos de género y una redistribución más equitativa de las tareas domésticas y de cuidado, estas labores siguen recayendo principalmente en las mujeres. Tanto es así que estimamos que el trabajo a domicilio es, en definitiva, funcional a la sostenibilidad del ejercicio del cuidado por parte de las mujeres. A su vez, la sujeción a la reproducción y el cuidado condiciona de manera persistente sus opciones laborales, lo que queda en evidencia en los casos de trabajadoras domiciliares estudiados.

\section{Agradecimientos}

Esta investigación forma parte de la tesis realizada en el Doctorado de Ciencias Sociales de la Universidad de Chile, en cotutela con el Doctorado de Sociología de la Universidad de São Paulo (USP), que contó con el apoyo de la Comisión de Investigación y Tecnología (CONICYT) y la International Network for Comparative Analysis of Social Inequalities (INCASI).

\section{Referencias bibliográficas}

Abramo, L.; Rodríguez, E.; Rossignotti, G. (2004). Guía educativa sobre cadenas productivas, trabajo a domicilio y organización sindical. Cuaderno del Facilitador. Lima: OIT.

Agacino, R.; Laire, F. de; Echeverría, M. (1993). La industria del cuero y el calzado después de una cadena. Desafios para un crecimiento con equidad. Santiago: PET.

Alonso, L.; Fernández Rodríguez, C. (2009). "Usos del trabajo y formas de la gobernabilidad: la precariedad como herramienta disciplinaria». En: SERRANO, A.; Crespo, E.; Prieto, C. Trabajo, subjetividad y ciudadanía, 229-258. Madrid: CIS.

Alvarez Newman, D. (2012). «Organización del trabajo y dispositivos de control en el sector automotriz: el toyotismo como sistema complejo de racionalización». Trabajo y Sociedad, XV (18), 43-57.

Arteaga, A. M.; León, K. de; Rojas, T. (2003). Trabajo a domicilio: ¿opción u obligación? Santiago: OXFAM; Activa Consultores.

Bauman, S. (1999). Trabajo, consumismo y nuevos pobres. Barcelona: Gedisa Editorial.

Baylina, M. (1996). Trabajo industrial a domicilio, género y contexto regional en la España rural. Barcelona: Universidad Autónoma de Barcelona.

Borgeaud-Garciandía, N.; Lautier, B. (2014). «La personalización de la relación de dominación laboral: las obreras de las maquilas y las empleadas domésticas en América Latina». Revista Mexicana de Sociología, 89-113. Recuperado el 19 de marzo 
de 2020 de <http://www.scielo.org.mx/scielo.php?script=sci_arttext\&pid=S018825032014000100004\&lng=es\&tlng=es $>$.

Braverman, H. (1983). Trabajo y capital monopolista. México: Nuestro Tiempo, SA.

Brega, C.; Duran; G.; Narbona, K.; PÁez, A. (2017). Estudio del trabajo en domicilio en la cadena del vestuario en Chile y de la creación de organizaciones sindicales territoriales de las trabajadoras involucradas. Santiago: Fundación Sol-OIT.

Caffarena, E. (1924). El trabajo a domicilio. Santiago: Oficina del Trabajo.

Caggiano, S. (2014). «Desigualdades entrelazadas, luchas divergentes, migración e industria textil en Argentina». Revista CIDOB, 151-170.

CEPAL-OIT (2016). Coyuntura laboral en América Latina y el Caribe. Cadenas mundiales de suministro y empleo decente. Santiago: OIT.

Coriat, B. (2011). El taller y el cronómetro. Ensayo sobre el taylorismo, el fordismo y la producción en masa. México: Siglo XXI.

Domínguez, N.; Arnes, L.; Torricella, P. (2012). «Desplazamientos teóricos, trayectos institucionales». La interseccionalidad en Debate. Berlín.

EDWARDs, P. (1990). El conflicto en el trabajo. Un análisis materialista de las relaciones laborales en la empresa. Madrid: Ministerio de Trabajo y Seguridad Social.

Edwards, P.; Scullion, H. (1987). La organización social del conflicto laboral. Control y resistencia en la fábrica. Madrid: Ministerio de Trabajo y Seguridad Social.

EgAN, J. (2019). «Trabajo a domicilio y organización sindical. Antecedentes y actualidad en torno al caso de las costureras argentinas». Izquierdas, 1-22. $<$ https://doi.org/10.4067/S0718-50492019000100001>.

Espinoza, G.; Soto, A. (2008). «El vínculo que establece el trabajador o trabajadora con la empresa flexible: el caso de una empresa de servicios». En: Soto, A. Flexibilidad laboral y subjetividades. Hacia una comprensión psicosocial del empleo contemporáneo, 279-309. Santiago: Lom.

GArZA, E. de la (2001). "La epistemología crítica y el concepto de configuración: Alternativas a la estructura y función estándar de la teoría». Revista Mexicana de Sociología. <https://doi.org/10.2307/3541203>.

- (2010). Hacia un concepto ampliado del trabajo: Del trabajo clásico al no clásico. México: UAM.

- (2011). "La revitalización del debate del proceso de trabajo». Revista Latinoamericana de Estudos do Trabalho, 7-35.

Godoy, L.; Stecher, A. (2008). "La experiencia de mujeres asalariadas en Santiago de Chile: sentidos del trabajo e identidades de género». En: EspinozA, B. Mundos del trabajo: pluralidad y transformaciones contemporáneas, 195-212. Quito: FLACSO-Ecuador.

Henríquez, H.; Riquelme, V. «El trabajo a domicilio, un tema antiguo y actual». Temas Laborales, 9. Santiago, Chile.

Henríquez, H.; Selamé, T.; Gálvez, T.; Riquelme, V. (1998). El trabajo a domicilio en Chile, un tema antiguo y actual: Resultados de una medición nacional. Cuestiones de desarrollo. Santiago: OIT.

Henríquez, H.; Selame, T.; Cardenas, A. (2005). Trabajo a domicilio en el siglo XXI. Tres miradas sobre el teletrabajo. Santiago: Dirección del Trabajo.

Kabat, M.; Pascucci, S. (2010). «El trabajo a domicilio como empleo precario. Alcances y límites de la legislación que intentó regularlo en la Argentina». VI Jornadas de Sociología de la UNPL. La Plata.

Katz, C. (2000). «La Teoría del Control Patronal: Balance de una discusión». Estudios del Trabajo, 3-34. 
Leite, M. (2004). «Tecendo a precarização: Trabalho a domicilio e estrategias sindicais na industria de confecção em São Paulo». Trabalho, Educação e Saúde, 239-265. <https://doi.org/10.1590/S1981-77462004000100005>.

Leite, M.; Alves, S.; Carbalho, P. (2017). «O trabalho na confeccão em São Paulo: as novas formas da precariedad». Caderno CRH, 30 (79), 51-68. <https://doi.org/10.1590/s0103-49792017000100004>.

Martínez Galarraga, J. La Revolución Industrial. Fundación Universitat Oberta de Catalunya.

Martini, G. (2001). De la Regulación a la desregulación del mercado laboral: el impacto socioeconómico de la flexibilización en el sector textil chileno. Madrid: UCM.

MarX, K.; Engels, F. (2002). El capital (vol. 1). Siglo XXI.

Maza, O. (2004). «El trabajo, una nueva lectura desde los procesos de precarización». Polis: Investigación y Análisis Sociopolítico y Psicosocial, 2 (4), 91-112.

Mello e Silva, L. (2014). «Braverman e a centralidade do proceso de trabalho: comentário sobre comentadores desde uma leitura periférica». Revista Brasileira de Sociologia, 150-182. <https://doi.org/10.20336/rbs.81>.

Menéndez, V. (2015). «El control y la resistencia en los procesos de trabajo: El caso de una empresa del sector forestal celulósico en Uruguay». Revista de Ciencias Sociales, 81-102.

Montero, C. (2000). La formación de capital humano en empleos atípicos: el caso del trabajo a domicilio. Santiago: CEPAL y GTZ.

Narbona, K. (2012). "La producción de compromiso en la empresa flexible, significados que los trabajadores dan al compromiso subjetivo con el trabajo, frente a las nuevas técnicas de gestión de recursos humanos. Estudio de caso de un banco multinacional en Santiago de Chile». Tesis de pregrado. Santiago.

Narotzky, S. (1988). Trabajar en familia: mujeres, hogares y talleres. Valencia: Edicions Alfons el Magnànim.

- (1997). "Cultura, región y trabajo en la Vega Baja del Segura. Revista Andaluza de Relaciones Laborales, 89-107. <https://doi.org/10.33776/trabajo.v3i0.234>.

OIT (2001). Seminario regional trabalho a domicilio e cadeias produtivas na América Latina: Desafíos para Acao Sindical. São Paulo.

Palacios, R.; Levy, I.; Urbina, D. (2015). «Identidad y trabajo informal a domicilio: la experiencia de cuatro mujeres chilenas». Batey: Revista Cubana de Antropología Sociocultural, 91-100.

Prates, S. (1984). «La mujer en el sector informal: las trabajadoras domiciliarias en la manufactura del calzado (Uruguay)». En: CEPAL. La mujer en el sector popular urbano. Santiago: Naciones Unidas.

Przeworski, A. (1988). Capital y socialdemocracia. Madrid: Alianza Editorial.

ReInecke, G. (1997). Flexibilidad, innovaciones y cadenas productivas: Industria textil $y$ del vestuario en Chile. Santiago: OIT.

RoldÁn, M. (1992). «La generización del proceso de trabajo y reestructuración industrial en los 90». Estudios del Trabajo, 85-124.

Salgado, P.; Carpio, J. (2017). "Superexplotación, informalidad y precariedad. Reflexiones a partir del trabajo en la industria de la confección». Estudios del Trabajo (54), 55-89.

Scotт, J. (2008). Género e Historia. México: FCE. 
Sevilhano Martínez, T. (2008). «Trabalho Domiciliar Feminino no Brasil: Determinantes familiares e productivos do trabalho remunerado exercido no propio domicilio». São Paulo.

<https://doi.org/10.11606/d.12.2008.tde-12052008-125836>.

Todaro, R.; YÁñez, S. (2004). El trabajo se transforma. Relación de producción y relaciones de género. Santiago: CEM.

Tomei, M. (1999). El trabajo a domicilio en paises seleccionados de América Latina: una visión comparativa. Santiago: OIT.

Valles, M. (1999). Técnicas cualitativas de investigación social. Reflexión metodológica $y$ práctica profesional. Madrid: Síntesis.

Zapata, M.; Sabina, P.; Chan, J. (2013). La interseccionalidad en debate. Berlín. 